\title{
Knowledge base concept for designing and documenting participation in radiological protection
}

\author{
T. Duranova ${ }^{1, *}$, C. Turcanu ${ }^{2}$, R. Geysmans ${ }^{2}$, C. Schieber ${ }^{3}$, C. Pölzl-Viol ${ }^{4}$, N. Zeleznik ${ }^{5}$, F. Barazza $^{6}$, \\ S. Economides ${ }^{7}$ and C. Fallon ${ }^{8}$ \\ ${ }^{1}$ VUJE - VUJE, a.s., 91864 Trnava, Slovak Republic. \\ 2 SCK-CEN - Belgian Nuclear Research Centre, Mol, Belgium. \\ ${ }^{3}$ CEPN - Centre d'étude sur l'évaluation de la protection dans le domaine nucléaire, Fontenay-aux-Roses, France. \\ ${ }^{4}$ BfS - Federal Office for Radiation Protection, Salzgitter, Germany. \\ ${ }^{5}$ EIMV - Milan Vidmar Electric Power Research Institute, Ljubljana, Slovenia. \\ ${ }^{6}$ EEAE - Greek Atomic Energy Commission, Athens, Greece. \\ ${ }^{7}$ FOPH - Federal Office of Public Health, Bern, Switzerland. \\ ${ }^{8}$ ULiège - University of Liège, Liège, Belgium.
}

\begin{abstract}
The stronger requirements for, and recognition of, stakeholder involvement in radiation protection, and the remaining challenges in translating these into practice, substantiate the need for knowledge sharing through a repository of stakeholder engagement experiences. One of the goals of the ENGAGE project was designing such a knowledge base and exemplifying it with case studies. Existing databases for stakeholder engagement were examined and combined with the focal points of the ENGAGE project. This paper presents the concept of the knowledge base by introducing its structure, which is then illustrated by the radon case studies developed within the ENGAGE project.
\end{abstract}

Keywords: knowledge base / stakeholder engagement / indoor radon / ENGAGE

\section{Introduction}

Within ENhancinG stAkeholder participation in the GovernancE of radiological risks for improved radiation protection and informed decision-making (ENGAGE) project, specific attention was given to the conceptualisation of stakeholders and stakeholder engagement; the rationales for and expectations from participatory processes; the level of engagement (e.g. with respect to the impact on policymaking); the participatory methods (e.g. workshops, focus groups, surveys, panels and others); and the consideration of both institutional and non-institutional forms of participation. Based on those results a design concept for a knowledge base that can contribute to learn on past experience was developed.

The ENGAGE project built on existing initiatives regarding the development of similar knowledge bases. With respect to emergency preparedness, response and recovery (EPR\&R), processes and forms of stakeholder engagement have been identified and documented already under the NERIS

\footnotetext{
* Corresponding author: tatiana.duranova@vuje.sk
}

European platform on preparedness for nuclear EPR\&R and other projects in this area. For instance, the EURANOS handbooks were designed and have been used as part of a participatory process involving the potentially affected stakeholders (Raskob et al., 2010). This led to the development of a proposal for building a knowledge base reporting on stakeholder workshops and public participation activities (French et al., 2014). As Janssens (2013) points out "people look across borders for information and guidance and want national measures to be subject to peer review by other countries or by international bodies" (p. S25). This need was especially evident after the Fukushima nuclear accident and the implementation of EU Basic Safety Standards (EU, 2013) and further justifies the activities undertaken in the knowledge base development.

Another example is the Organisation for Economic Cooperation and Development (OECD) pilot database on stakeholder engagement practices, which provides examples of stakeholder engagement in regulatory policies of OECD member and partner countries. This database gives a general perspective on how such knowledge could be collected and documented for further use (OECD, 2016). 
The review of existing knowledge base structures aimed at identifying how these databases can be extended or improved in order to include the key research questions addressed in the ENGAGE project. Round tables discussions with radiation protection researchers and practitioners at the NERIS 2018 workshop (Duranova et al., 2018) formed the basis for further reflection. The concept of knowledge base aims at documenting participatory practices taking into account the specific aspects of three types of radiation exposure: medical, indoor radon and emergency/post-accident. For example, the approaches to develop a radiological protection culture in the field of emergency preparedness, response and recovery were compared and contrasted with similar approaches developed for medical and indoor radon issues (Barazza et al., 2019).

This paper provides the concept of a knowledge base that emerged from the analyses and the case studies.

\section{ENGAGE knowledge base concept}

The analysis and the case studies within the ENGAGE project were driven by the following general questions:

- How are radiation protection (RP) communities responding to "external" pressures, mandates, demands, and/or expectations that emerged in public venues commending the engagement of stakeholder and how does this show in practice (e.g. specific cases)?

- Which real or potential forms of stakeholder engagement can be observed in RP practice, showing no reference to existing requirements?

- What is the potential role of radiological protection culture for enhancing stakeholder engagement and informed decision-making?

These questions were addressed in the wide range of case studies conducted in the ENGAGE project and documented in a number of project reports (Turcanu et al., 2019a, 2019b; Zeleznik et al., 2019; Barazza et al., 2019).

The analysis of existing knowledge bases documenting stakeholder engagement demonstrated that for practical reasons it is better to have a simple knowledge base structure. The concept proposed should simultaneously allow for broader views on engagement, and reflection of the different forms of, and motivations for, engagement. The concept should offer inspiration also for stakeholders other than radiation protection experts, and for other forms of engagement.

Information on the participatory event/practice/process is collected under the database structure given below. The database form consists of seven sections with the following information about the event/practice/process:

- "Provider of information": short data about the one who is providing the information;

- "General description": details about the context of the activity (e.g. objectives, context, initiator, organiser, funding body);
- "Participants": information about opportunities and motivations for participation;

- "Process": description of participation with forms and methods, process evaluation, flexibility, ethical considerations;

- "Ex-post assessment": evaluation of the process (e.g. challenges, outcome, feedback from participants);

- "Practical information": timeline, facilities and resources;

- "Additional information" and other sources (e.g. links to reports).

For each of the items the driving questions, explanations and some examples are given to further describe and specify what kind of information is expected (Duranova et al., 2019).

\section{ENGAGE knowledge base implementation: radon case study}

The knowledge base concept was illustrated with a number of case studies conducted within the ENGAGE project (Duranova et al., 2019). They have been related to the area of medical exposure, exposure to indoor radon and emergency and post-accident exposure.

For illustration, in Table 1, we introduce the case study from Switzerland documenting the process of actions undertaken in the framework of the implementation of the radon national action plan 2012-2020.

\section{Conclusions}

Further steps in the development of the knowledge base could comprise of operational realisation and implementation of the ENGAGE knowledge base in practice.

As proposed also by French et al. (2014), the knowledge base on stakeholder engagement in radiation protection could be hosted on a web-server using a database/knowledge management system. Users would be able to share their experiences with NERIS, SHARE and other research platforms and interested communities, and access other published reports. An electronic database or web-based tool would allow end-users to consult the database or contribute to its further development and maintenance. Good search functions will be necessary to allow selection of relevant case studies.

Further development of the knowledge base to make it operational could be a part of the joint road map for radiation protection research (Impens and Salomaa, 2020).

Acknowledgements. The work described in this paper was conducted within the ENGAGE project, which was part of the H2020 CONCERT project. This project received funding from the Euratom research and training programme 2014-2018 under grant agreement No. 662287.

Disclaimer (Art. 29.5 GA). This publication reflects only the author's view. Responsibility for the information and views expressed therein lies entirely with the authors. The European Commission is not responsible for any use that may be made of the information it contains. 
Table 1. ENGAGE knowledge base: exposure to indoor radon case study.

\author{
Provider of information \\ Organisation \\ Name (optional) \\ E-mail or other contact details \\ General description \\ Title \\ Country \\ Exposure situation
}

Objective of the participatory practice

\section{Broader context}

\section{Organisation}

Participants

Opportunities for participation

\author{
Motivations for participating \\ Process \\ Participation forms and methods
}

Process evaluation

Flexibility

Ethical considerations

Ex-post assessment

Actual outcomes

\section{Outcome evaluation}

Key strengths

Challenges

Feedback

Practical information

Timeline of event (including preparation The action plan is in progress from 2012 to 2020. The revised ordinance took effect in 2018 and planning)

Facilities used

Financial resources

Human resources

Detailed information
Federal Office of Public Health, Switzerland

Fabio Barazza

fabio.barazza@bag.admin.ch

Actions undertaken in the framework of the implementation of the radon national action plan 2012-2020

Switzerland

$\square$ Medical exposure

$\mathrm{X}$ Exposure to indoor radon

Emergency and post-accident

Other

Main goals are the promotion of protective measures against radon in buildings, planning an efficiency strategy for remediation, including radon in the training of construction experts and improving public awareness to health problems caused by radon

Switzerland is strongly affected by the radon problem. It is clear that besides a legal framework, additional actions are needed and therefore a national action plan was developed All actions in the context of the radon problem are based on corresponding regulations included in the ordinance on radiation protection since 1994

It is planned to introduce a new action plan in 2021

The Swiss national radon action plan was developed, implemented and funded by the Federal Office of Public Health

Most important partners for the implementation of the action plan were the local authorities (cantons). In addition, many universities, in particular of applied sciences, as well as other federal organizations were involved. Overall, there was a strong willingness to participate and participation was open to all interested parties

All-important partners for the implementation of the action plan were motivated to participate

Conferences

Workshops

Information campaigns

Training courses

The process has not been evaluated

The main goals of the action plan were defined in collaboration with the local authorities (cantons). They were our most important partners. The aims were not adapted during the process

Ethical aspects such as transparency, equality, fairness, and collaboration are considered in all activities of the Federal Office of Public Health

The main outcome is a new legal framework for the protection against radon laid out in the revised ordinance on radiation protection. In addition, new measurements protocols, training courses for radon experts, and teaching material for the formation of different professional within the construction sector were introduced. The role and duties of recognized measuring services were clarified. The radon map and the radon database were developed further The outcomes of the action plan have been evaluated by an external evaluator. The corresponding recommendations constitute an important input for the development of the future strategy concerning the protection against radon

The participation and involvement of all relevant partners in the process of improving the protection against radon construction experts could not always be convinced that the radon problem is important. All activities and outcomes of the action plan have been published and made available
The public and many members of the construction sector such as architects and other
No special facilities were used

The financial resources used were justified in view of the achieved results

Cannot be quantified www.ch-radon.ch 


\section{References}

Barazza F, Cardis E, Cantone M-C, Charron S, Doremus P, Duranova T, Economides S, Gschwind R, Lafage S, Liutsko L, Murith C, Schieber C, Schneider T. 2019. Final report on case studies, including recommendations and guidelines on building and enhancing radiation protection culture. CONCERT Deliverable D9.87.

Duranova T, Bohunova J, Turcanu C, Abelshausen B, Zeleznik N, Pölzl-Viol C, Schieber C. 2018. Knowledge base for stakeholder engagement in radiation protection. In: Fourth NERIS Workshop, 25-27 April 2018, Dublin, Ireland.

Duranova T, Pölzl-Viol C, Turcanu C, Geysmans R, Abelshausen B, Schieber C, Croüail P, Zeleznik N, Economides S, Barazza F, Fallon C. 2019. Knowledge base for designing and documenting stakeholder engagement process. CONCERT Deliverable D9.92.

EU. 2013. Council directive 2013/59/EURATOM of 5 December 2013 laying down basic safety standards for protection against the dangers arising from exposure to ionising radiation.

French S, Schneider T, Croteau C, Oughton D, Tomkiv Y, Papamichail N. 2014. Designing and reporting stakeholder workshops and public participation: proposal for a building $a$ knowledge base. NERIS Platform report, Version 6.0.

Impens N, Salomaa S. 2020. Second road map for radiation protection research. CONCERT Deliverable D3.7.
Janssens A. 2013. EU basic safety standards and European response to the Fukushima accident. Radioprotection 48(5): S19-S26.

OECD. 2016. Pilot database on stakeholder engagement practices. Measuring Regulatory Performance Programme. OECD. Available from: http://www.oecd.org/gov/regulatory-policy/pilot-data base-on-stakeholder-engagement-practices.htm.

Raskob W, Gering F, Lochard J, Nisbet A, Starostova V, Tomic B. 2010. Overview and main achievements of the EURANOS project: European approach to nuclear and radiological emergency management and rehabilitation strategies. Radioprotection 45(5): S9-S22.

Turcanu C, Pölzl-Viol C, Fallon C, Perko T, Cantone M-C, Zeleznik N. 2019a. Venues, challenges, opportunities and recommendations for stakeholder engagement in relation to indoor radon exposure. CONCERT Deliverable D9.91.

Turcanu C, Abelshausen B, Geysmans R, Van Oudheusden M, Meskens G, Schieber C, Schneider T, Zeleznik N, Pölzl-Viol C, Perko T, Fallon C, Duranova T, Cantone M-C, Murith C, Barazza F, Economides S, Liutsko L, Gschwind R, Charron S, Savu D. 2019b. Final report of the ENGAGE project. CONCERT Deliverable D9.94.

Zeleznik N, Pölzl-Viol C, Geysmans R, Turcanu C, Abelshausen B, Schieber C, Duranova T, Zorko B, Liutsko L. 2019. Venues, challenges, opportunities and recommendations for stakeholder engagement in emergency \& recovery preparedness and response. CONCERT Deliverable D9.90.

Cite this article as: Duranova T, Turcanu C, Geysmans R, Schieber C, Pölzl-Viol C, Zeleznik N, Barazza F, Economides S, Fallon C. 2020.

Knowledge base concept for designing and documenting participation in radiological protection. Radioprotection 55(HS2): S255-S258 